Julia Sanmartin (ed.)

Discurso turístico e Internet

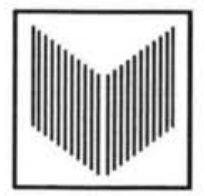


Este libro ha sido publicado gracias a la ayuda económica del proyecto precompetitivo "Implementación y explotación léxica del corpus turistico multilingüe de la Comunidad Valenciana", concedido por la Universitat de València en 2010.

Los autores de cada articulo son los responsables exclusivos de su contenido.

Reservados todos los derechos

(C) Iberoamericana, 2012

Amor de Dios, 1 - E-28014 Madrid

Tel.: +34914293522

Fax: +34914295397

info@iberoamericanalibros.com

www.ibero-americana.net

C Vervuert, 2012

Elisabethenstr. 3-9 - D-60594 Frankfurt am Main

Tel.: +49695974617

Fax: +49695978743

info@iberoamericanalibros.com

www.ibero-americana.net

ISBN 978-84-8489-614-2 (Iberoamericana)

ISBN 978-3-86527-668-1 (Vervuert)

Depósito Legal: SE-332-2012

Diseño de la cubierta: Carlos Zamora

Impreso en España por Publidisa

Este libro está impreso integramente en papel ecológico blanqueado sin cloro 


\title{
PÁGINAS WEB PRIVADAS E INSTITUCIONALES: \\ EL USO DE LA ADJETIVACIÓN \\ EN UN CORPUS INGLÉS-ESPAÑOL \\ DE PROMOCIÓN DE DESTINOS TURÍSTICOS
}

\author{
NuRIa Edo MARZÁ \\ IULMA-Universitat Jaume I
}

\section{La industria del turismo: un "gigante" muy particular}

El turismo como tal nace en el siglo XIX a raíz de la Revolución Industrial y del incremento que esta supuso en el número de desplazamientos cuya intención principal era el ocio, el descanso, la cultura, la salud, los negocios o las relaciones familiares.

El Diccionario de la lengua española de la Real Academia define la unidad léxica (UL) turismo como: "la actividad o hecho de viajar por placer; el conjunto de los medios conducentes a facilitar estos viajes; $y$ el conjunto de personas que realiza este tipo de viajes" (RAE 2010).

El análisis del turismo como conjunto o agrupación de los tres significados aducidos arriba es una tarea harto compleja en tanto en cuanto las características y el número de participantes, tipologías, situaciones, agentes, objetivos, contextos y otros parámetros a tener en consideración a la hora de caracterizar esta industria o sector en su conjunto escapan, en la mayoría de los casos, a una caracterización clara y estanca, bien definida o fácil de elaborar.

Por ejemplo, la tipología de productos turísticos es amplia y variada, y pueden encontrarse cientos de clasificaciones de estos productos. A nivel muy general y dejando de lado tipos de turismo muy específicos (como el turismo espacial), podríamos enumerar dentro de esta tipología el turismo de masas (el más convencional y normalmente asociado al turismo de sol y playa), el turismo individual (en el que no intervienen los operadores turísticos), el turismo cultural (dentro del cual encontraríamos el turismo monumental, urbano, arqueológico, de compras, literario, gastronómico, etc.), el turismo natural (como el turismo rural, micológico o el agroecoturismo entre mucho otros), el turismo activo (como el turismo de aventura o el turismo termal o de salud entre otros) y el turismo de negocios.

En cuanto a los participantes, existen dos tipos básicos dentro de la comunidad discursiva de la industria del turismo: los expertos en el área (especialistas en la materia y agentes de la gestión de esta industria) y los legos o no expertos 
en el área (los turistas en sí) (Edo Marzá 2010). A los primeros (expertos) los podriamos considerar agentes o participantes "constantes", porque siempre forman parte de la comunidad discursiva turística, y a los segundos (los turistas), participantes "circunstanciales" en dicha comunidad, porque entran y salen de la misma en función de la actividad turística realizada (al viajar o dejar de hacerlo). Entre los expertos encontramos, por ejemplo, a los guías y operadores turísticos, a los empleados de las agencias de viajes, a las personas que trabajan de cara al público en hoteles y diferentes espectáculos turísticos, etc.

Además, la gran cantidad de temas interrelacionados que forman parte de lo que se entiende por "turismo", esto es, alojamiento, recreo, gestión del destino turistico y patrimonio entre otros (Balboni 1989), otorgan a la industria del turismo una rica naturaleza multidimensional e incluso multidisciplinar.

Pese a o, quizá, debido a esta complejidad inherente a la industria del turismo -que la hace cada vez más variada y atractiva-, el sector turístico es una pieza clave en la economía de muchos países, algunos de los cuales sobreviven prácticamente de manera exclusiva de los ingresos que les reporta dicho sector. De hecho, el turismo es la primera industria a nivel mundial. No obstante, se trata de un sector frágil en muchos sentidos, puesto que suele mostrar un comportamiento fluctuante, irremediablemente unido a los ritmos económicos de las naciones y al comportamiento de las economías mundiales. Cuando no hay dinero, a lo primero que se renuncia suele ser al ocio. Por ello, a la hora de "engrasar" el engranaje del turismo y hacerlo funcionar a buen ritmo, es fundamental dar a conocer y "vender" el destino turístico de manera efectiva y atrayente para el turista potencial. En este sentido, los conceptos de destino y márquetin -así como sus fundamentos- juegan un papel primordial que aumenta su capacidad de atracción al conjugarse ambos; el rol crucial del primero (destino) dentro de la industria del turismo lo reconocen numerosos autores como Ryan y Cave:

Given the primacy of "destination" in tourism as the away-from-home locale wherein attractions and activities are located, it is not surprising that a significant component of the academic tourism literature has been devoted to "the destination" (2005: 143).

En este contexto, la importancia de la mercadotecnia o márquetin deriva de la necesidad que el destino turístico tiene de estas estrategias de mercado: el márquetin de los destinos turísticos es una pieza clave de la industria turística al representar el "conjunto de principios y prácticas que buscan el aumento del comercio, especialmente de la demanda", así como el "estudio de los procedimientos y recursos tendentes a este fin" (RAE 2010), algo fundamental a la hora de promocionar de manera efectiva un destino. 
Como señalan Bigné, Font y Andreu,"tradicionalmente, el marketing de los destinos tenía como finalidad incrementar el número de visitas, considerando el turismo como cualquier otro producto" (2000: 32 ). Sin embargo, tal y como apuntan estos mismos autores, no se debe identificar el márquetin de destinos únicamente con promoción del destino, sino con la satisfacción de las necesidades de los turistas y la comunidad local. Es decir, una vez atraído el cliente, habrá que intentar que permanezca en el destino y que lo haga cumpliendo todas sus expectativas de manera que sea el germen del márquetin más eficaz que existe: el popular "boca a boca". Cuando "vendemos" (promocionamos) un destino turístico y en general cualquier "producto" vendible, lo que buscamos principalmente es atraer la atención del potencial comprador tratando de convencerle de que dicho "producto" satisfará sus necesidades y deseos; intentamos llegar al mayor número de personas, a un público cuanto más amplio, mejor. Buscamos aumentar en lo posible la demanda del producto, procurando alcanzar altos niveles de satisfacción en el cliente, que será, si queda complacido, nuestro mejor elemento promocional.

Es por todo lo comentado hasta el momento, por sus particularidades y objetivos, que la industria del turismo busca la plataforma de promoción que mejor se adecúe a sus necesidades en cada momento, habiendo encontrado en Internet un aliado imprescindible.

\section{El turismo y las nuevas tecnologías: el uso de las páginas electrónicas con fines promocionales}

Como veremos en esta sección y partiendo de lo comentado en el apartado anterior, el uso de las Tecnologías de la Información y la Comunicación (TIC) es hoy en día un aspecto fundamental en la industria del turismo en general y en la promoción de los destinos turísticos en particular:

El turismo es o puede ser considerado una industria estrechamente vinculada con los sistemas de información, sobre todo si tenemos en cuenta que hablamos de una extensa industria donde sus productos/servicios son bienes intangibles y que precisan de una información fidedigna, actualizada, amplia y detallada para su promoción y, sobre todo, para su óptima comercialización (Majó/Gali 2002: 397-398).

Sin embargo, siendo un poco más específicos, no es solo la industria del turismo la que puede beneficiarse de la informática sino que también esta última necesita del turismo; tal y como apunta Álvarez (1998: 135), dos hechos permiten pensar en una estrecha interrelación entre la industria turística y la informática: el primero, que la información está en los mismos cimientos de la industria 
turística y es parte fundamental de la misma y, el segundo, que el turismo, al ser la primera industria mundial, se convierte potencialmente en el principal cliente de la industria informática.

En este estudio vamos a centrarnos en un cibergénero o en el que muchos consideran "el cibergénero" por excelencia: las páginas web o electrónicas, y más especificamente en las páginas web promocionales de destinos turísticos. Con un corpus de páginas web de este tipo analizaremos y compararemos el uso de la adjetivación tanto en español como en inglés en dichos sitios web, pero para ello primero hay que clarificar ciertos aspectos sobre qué consideramos una página web, qué debe contener una Web de calidad y por qué o de qué manera son importantes para la promoción de los destinos. En general, se podría decir que una página web es un documento electrónico que se halla en una red informática (World Wide Web), al que se accede mediante enlaces de hipertexto y que generalmente forma parte de un sitio web. Calvi (2010) considera las páginas web como un ejemplo de macrogénero, esto es, un producto tangible, identificable sobre todo por el emisor y el canal utilizado, así como por un propósito dominante, pero caracterizado a su vez por la hibridación de diferentes géneros, tipologías textuales, estilos, etc. Del mismo modo, describe la página web dentro del ámbito turístico como un macrogénero cuyo propósito dominante suele ser el promocional y que puede contener guías descriptivas, guías prácticas, secciones reservadas a los profesionales (con foros, normativas, etc.), foros de viajeros, blogs, etc. Estas páginas web pueden ser emitidas por instituciones nacionales, autonómicas, municipales, por organizaciones comerciales o por comunidades de viajeros. El hecho de que la página web sea un macrogénero, afirmación con la que estamos totalmente de acuerdo, no viene sino a aumentar la complejidad inherente a la industria turística, pero también la enriquece sobremanera por el abanico de posibilidades que se abren a la hora de promocionar los diferentes destinos turísticos.

Hasta hace unos años, el proceso de promoción turística se realizaba a través de los folletos tradicionales, que debían estar dirigidos a mercados potenciales específicos. Con la aparición de Internet, nos encontramos frente a un mercado mucho más global (el de los usuarios de Internet), en el que no hace falta dar un folleto en mano para que llegue a alguien, sino que ese alguien pueda encontrar la información que busca con tan solo una dirección web o un mensaje de búsqueda correcto y un "click".

The tourism industry is a global Enterprise that has captured the relevance of the web as a new mass medium for contacting potential receivers all over the world and promoting tourist products both in domestic and international markets (Pierini 2009: 95).

Así pues, además de tener el potencial de poder llegar a un número infinitamente superior de usuarios y de poder ser mucho más perdurable que el folleto 
tradicional (teniendo un uso indefinido en el tiempo), la promoción a través de Internet, y más concretamente a través de páginas web puede ser actualizada periódicamente e incluso adaptada al perfil de cada usuario, tiene un coste menor que el folleto, permite recopilar una mayor cantidad de información, especialmente de tipo visual, y ofrece una inmediatez difícil de conseguir con otros medios o soportes. Sin olvidar que, al tratarse de un macrogénero, puede incluir otros cibergéneros complementarios e interactivos como pequeños foros de opinión. Por tanto, hemos elegido las páginas web promocionales como género o macrogénero para estudiar la adjetivación por el potencial que representan, por su uso cada vez más extendido y demandado, por su inmediatez y accesibilidad. y por ser lo que consideramos el presente y el futuro de la información turística.

Teniendo en cuenta este objetivo promocional que pretendemos analizar desde la perspectiva de los adjetivos utilizados, no es dificil deducir que la regla de oro a la hora de diseñar y construir cualquier página web es que la navegación sea clara y sencilla para que la información llegue de manera directa y sin ambages. Para ello, el texto en las páginas web debe invitar a leer, por lo que toda "buena Web" debe evitar los textos pequeños o poco legibles. La información debe ser clara, representativa y relevante. Además, deben cuidarse los pequeños detalles de manera que la Web sea un reflejo del buen servicio ofrecido. Los colores y las fuentes deben estar elegidos con acierto y deben evitarse las imágenes superfluas porque, pese a la importancia del contenido visual, este debe tener sentido y no "empalagar". Las páginas deben poder imprimirse correctamente y se debe facilitar el contacto vía email con los responsables de la Web. Deben evitarse por todos los medios los errores ortográficos y gramaticales. (WebTaller.com 2010). En cada Web, la información debe ser pertinente, veraz, adecuada y no superflua y el estilo debe ser capaz de motivar a seguir leyendo. Conocer las características básicas de una buena Web ha sido fundamental en este estudio, especialmente a la hora de seleccionar las páginas que posteriormente han formado parte de nuestro corpus. Los arriba mencionados han sido algunos de los parámetros o criterios adoptados a la hora de seleccionar las páginas web que se analizan en este estudio y las claves a la hora de entender de qué manera, además de mediante el mero uso de adjetivos, una página web promocional de destino turístico en su conjunto cumple con su misión.

\section{La importancia del lenguaje en la promoción de los destinos turísticos: la adjetivación}

Como se deduce de los dos apartados anteriores, las estrategias de mercadotecnia tienen indudablemente un componente visual que resulta crucial y determi- 
nante, máxime cuando nos referimos a la promoción de los destinos turísticos. Tal y como hemos visto en la sección 2, las páginas web no se concebirían sin el papel fundamental que juega la imagen en ellas. No obstante, el lenguaje juega igualmente un papel fundamental a la hora de atraer al turista a través de una página web, porque puede ser tanto o más concreto e informativo que la imagen en muchos casos y, sobre todo, porque ahorra espacio en la página web. En palabras de Majó y Gali: "podemos definir la información turística como el conjunto de servicios que se ofrecen al turista con el objetivo de informarlo y orientarlo durante su estancia, o incluso, todas aquellas informaciones que le ayudarán a prepararlo de forma más precisa" (2002: 397). El turismo está irremediable y necesariamente ligado a la información y a la correcta transmisión y recepción de la misma, ya sea a través de la imagen o del lenguaje. Una de las claves del turismo radica en crear, alimentar o incrementar el interés del turista por descubrir lugares nuevos y nuevas vivencias, y es esto precisamente lo que esta industria trata de mantener vivo, fomentar y promocionar a la hora de "vender" destinos. El turista quiere descubrir pero para ello necesita contar de antemano con ciertas garantías y tener la información necesaria para cerciorarse de que su experiencia turística será placentera.

Cuando un usuario entra en la página web de un hotel para efectuar una reserva, no solo le interesa (y mucho) ver imágenes reales de las habitaciones en las que se hospedará, el comedor..., sino también conocer características concretas (tarifas, posibilidad de añadir camas supletorias, accesibilidad a una conexión wi-fi, etc.) que solo pueden explicitarse mediante el lenguaje. Pero aún hay más, puesto que junto con la información objetiva de las características del destino, todas las páginas web que promocionan algún destino turístico tienden a emplear párrafos verdaderamente plagados en algunos casos de adjetivos, con el fin de persuadir y "enamorar" mediante el lenguaje al turista potencial. Como apunta Pierini (2009), los adjetivos, utilizados tanto para modificar como para modular o elaborar el significado de los nombres, juegan un papel crucial en el discurso, ya que se usan para describir una entidad, poniendo de relieve y enfatizando alguna propiedad o caracteristica, o para expresar una opinión, un juicio o una actitud emocional hacia una entidad, teniendo el poder de condensar una evaluación en un único lexema.

Con el uso un tanto exagerado o hiperbólico de la adjetivación, a veces se corre el peligro de despertar en el usuario expectativas desmedidas, perspectivas demasiado optimistas que no favorecen a la industria si el cliente se siente en cierta medida defraudado o decepcionado al finalizar su experiencia turística en el destino. No hace falta hacer una búsqueda demasiado intensiva en Internet para darse cuenta del exhaustivo y a veces algo exagerado uso de la adjetivación en las páginas web. En el ejemplo que encontramos abajo, por ejemplo, y que ha sido 
extraído de nuestro corpus de estudio (ver apartado 5), comprobamos la profusión de adjetivos (en negrita en el texto) utilizados a la hora de promocionar un hotel:

Situado en la privilegiada zona de la costa Azahar, el hotel Marina d'Or 5 estrellas está ubicado en primera_línea de playa, cerca del resto de hoteles y apartamentos $[\ldots]$.

Dispone de 184 habitaciones, incluyendo espléndidas suites, suites junior y habitaciones adaptadas para minusválidos. Es un hotel moderno y funcional, con excelentes y exclusivas vistas al mar. Con un alto nivel de servicio y atención al cliente, ofrece una excelente oferta gastronómica en su restaurante buffet especializado en cocina mediterránea (Web Marina d'Or).

Del mismo modo, una descripción o presentación de un destino turístico demasiado "descafeinada" en una página web corre el peligro de desalentar al turista que busca información desde su ordenador y decepcionarlo antes siquiera visitado el destino. Si el turista prefiere que se ensalcen las bondades del destino turístico o que se presenten de manera objetiva, neutral y en tono meramente descriptivo, dependerá, suponemos, del tipo de turista, pero pese a lo que pueda parecer a simple vista, también es cierto que no es raro encontrar párrafos promocionales meramente descriptivos:

El Hotel Rural El Rosal es un establecimiento que viene prestando sus servicios de alojamiento y restauración desde 1992. A principios del año 2008 se realizó una profunda reforma en todas las estancias de mismo: habitaciones, cafetería, restaurante (Web de hoteles Muchoviaje.com).

El uso más o menos profuso de adjetivos, la descripción más o menos exagerada de los aspectos positivos de un destino y la calidad de la información turística en general tendrán siempre un eminente sentido subjetivo, pues son muchas las variables que los condicionan y variadas son también las preferencias del turista potencial. Así pues, entre estas variables a "premiar" (valorar positivamente) o "castigar" (valorar negativamente) por el turista encontramos, según Majó y Galí (2002), las siguientes:

- La relevancia o adecuación de la información según lo solicitado;

- La exhaustividad y detalle, por exceso o por defecto, de la información obtenida;

- La focalización y precisión;

- La adecuación temporal entre la obtención de la información y las necesidades del turista;

- Finalmente, el formato con que se presenta la información. 
El uso de la adjetivación incidirá principalmente en los tres primeros puntos mencionados arriba y contribuirá, por tanto, a que el turista potencial considere, o no, la información proporcionada relevante, adecuada y detallada en su justa medida. El uso de la adjetivación tanto por exceso como por defecto afectará de forma distinta a la percepción del turista y podrá desalentarle desde el primer momento, agradarle y por tanto captarle o resultarle un ejercicio empalagoso en el que solo se le trata de "vender humo".

Para analizar la adjetivación en las páginas web promocionales de destinos turísticos, en este artículo partiremos de la base de que el lenguaje del turismo puede ser considerado como una lengua de especialidad debido a una serie de razones que en él se cumplen, tales como: la existencia de géneros propios (guias de viajes, revistas de viajes y turismo, catálogos de viajes, folletos de hoteles, páginas web, billetes, reservas, etc.), el hecho de tener un carácter funcional para los objetivos comunicativos y el hecho de promover la creación de léxico especializado. De este lenguaje de especialidad nos centraremos, como ya hemos dicho, en el uso de la adjetivación, y trataremos de extraer conclusiones relevantes para entender mejor el uso de estas unidades léxicas tanto en inglés como en español en las páginas web promocionales de destinos turísticos. Antes de pasar al análisis propiamente dicho de estas unidades, el apartado 4 da cuenta de la metodología empleada, los objetivos perseguidos y el tipo de resultados a obtener en este estudio.

\section{Metodología de análisis: el corpus de estudio, herramientas de explotación y objetivos}

Este estudio y los resultados que de él se derivan se originan en el análisis de un corpus bilingüe (inglés y español), no anotado y compilado ad hoc de páginas web relacionadas con la industria del turismo y que tienen en común el hecho de ser páginas promocionales de algún destino turístico (incluyendo como tal la promoción del alojamiento en dichos destinos). Para ello, nos hemos centrado en las páginas web promocionales de diversas ciudades y lugares (destinos turísticos en sí), así como en los alojamientos (principalmente páginas web de hoteles) ofertados en dichos destinos.

El inicio real de este estudio se sitúa, sin embargo, en el proyecto COMET. VAL (Corpus Multilingüe en Turismo de Valencia), destinado a compilar un corpus multilingüe con el mismo nombre de textos promocionales web procedentes de fuentes institucionales del destino "ciudad de Valencia". Así pues, este corpus COMET.VAL se compiló, en origen, en una base de datos terminológica en Filemaker que contaba con registros en español, inglés, francés, alemán y catalán. 
Posteriormente, esta base de datos incorporó registros de otros muchos destinos turísticos alrededor del mundo asi como muestras representativas de todos los géneros textuales característicos de la industria del turismo -no solo de páginas web de fuentes institucionales- en español, inglés y francés. Asimismo, para la realización del presente estudio se ha procedido a compilar el corpus tomando muestras textuales no solo de COMET.VAL y de su posterior implementación, sino incorporando, además, muestras procedentes del corpus de estudio "Tánger" (corpus de gestión hotelera aún en desarrollo), que contiene básicamente información procedente de páginas web de hoteles.

Como ya se ha venido apuntando, el presente estudio pretende dar cuenta del uso de la adjetivación en las páginas web turísticas, principalmente las de hoteles y las de sitios web oficiales que promocionan destinos turísticos. Por ello, el hecho de haber compilado los corpus COMET.VAL (y su implementación) y "Tánger" en una base de datos en Filemaker nos ha permitido poder recuperar ahora y compilar en un corpus ad hoc (especialmente diseñado para la realización de este estudio) aquellas muestras textuales que queremos analizar: sitios web de promoción de destinos turísticos y páginas web de hoteles en español y en inglés, no considerando para este estudio otras muestras textuales de estos corpus pertenecientes a otros géneros. A esto hay que sumar una implementación propia llevada a cabo en el corpus de español por presentar este, en un principio, un número de palabras menor al del corpus inglés. Los datos cuantitativos y la composición del corpus de estudio pueden observarse en las Tablas 1 y 2 .

\section{TABLA 1}

Composición, contenido y número de palabras del corpus de estudio ESPAÑoL

\begin{tabular}{|l|l|c|}
\hline $\begin{array}{l}\text { Corpus de estudio } \\
\text { ESPAÑOL }\end{array}$ & \multicolumn{1}{|c|}{ Contenido } & $\begin{array}{c}\text { N } \\
\text { palabras }\end{array}$ \\
\hline $\begin{array}{l}\text { COMET.VAL y su } \\
\text { implementación }\end{array}$ & $\begin{array}{l}\text { Páginas web institucionales del destino } \\
\text { turístico ciudad de Valencia (español). } \\
\text { Páginas web (institucionales o no) de otros } \\
\text { destinos turísticos españoles o de habla } \\
\text { hispana. }\end{array}$ & 10945 \\
\hline Tánger & Páginas web de hoteles en español. & 110219 \\
\hline Implementación propia & $\begin{array}{l}\text { Páginas web (institucionales o no) de otros } \\
\text { destinos turisticos españoles. } \\
\text { Páginas web de hoteles en español. }\end{array}$ & $\mathbf{4 4 0 3 4}$ \\
\hline No total de palabras en español & $\mathbf{1 6 5}$ \\
\hline
\end{tabular}


TABLA 2

Composición, contenido y número de palabras del corpus de estudio INGLÉS

\begin{tabular}{|l|l|c|}
\hline $\begin{array}{l}\text { Corpus de estudio } \\
\text { INGLÉS }\end{array}$ & \multicolumn{1}{|c|}{ Contenido } & $\begin{array}{c}\mathbf{N}^{\circ} \text { de } \\
\text { palabras }\end{array}$ \\
\hline $\begin{array}{l}\text { COMET.VAL y su } \\
\text { implementación }\end{array}$ & $\begin{array}{l}\text { Páginas web institucionales del destino } \\
\text { turístico ciudad de Valencia (versión en } \\
\text { inglés). } \\
\text { Páginas web (institucionales o no) de otros } \\
\text { destinos turísticos de habla inglesa. }\end{array}$ & 16011 \\
\hline Tánger & Páginas web de hoteles en inglés. & 145438 \\
\hline $\mathbf{N}^{\circ}$ total de palabras en inglés & $\mathbf{1 6 1 4 4 9}$ \\
\hline
\end{tabular}

Una vez compilado el corpus de estudio bilingüe se ha procedido a la explotación, observación y análisis de los datos contenidos en él mediante el uso del programa WordSmith Tools 5.0 (WST); un programa de análisis de corpus textuales gracias al cual se pueden explotar grandes cantidades de texto mediante búsquedas de carácter léxico, (con)textual o estadístico.

En nuestro caso, el análisis llevado a cabo -en base al tipo de datos/resultados que queríamos obtener- ha sido el siguiente: en primer lugar, con la herramienta Wordlist de WST, hemos elaborado listados de frecuencia desprovistos de palabras gramaticales para observar la incidencia de las distintas categorías gramaticales en nuestro corpus - prestando una especial atención al lugar que ocupan los adjetivos y al peso específico de cada categoría en el corpus-. A continuación, hemos estudiado las unidades léxicas con mayor frecuencia de aparición, intentando extraer conclusiones significativas en cuanto a la composición del corpus o a los lexemas de uso frecuente y característico en los géneros web promocionales turísticos.

En segundo lugar, hemos analizado con mayor detalle los listados de frecuencia seleccionando los adjetivos que aparecen entre las 1000 palabras más frecuentes del corpus y hemos creado unas tablas que nos han permitido visualizar de manera conjunta los adjetivos más frecuentes en ambas lenguas de trabajo.

En tercer lugar, hemos clasificado los adjetivos según una tipología propia que nos ha permitido reconocer los tipos de adjetivos más utilizados con fines promocionales en las páginas web en ambas lenguas y las conclusiones que pueden derivarse de ello.

En cuarto lugar, mediante la herramienta Concord de WST y más específicamente sus aplicaciones "Concordancias" y "Colocados", hemos analizado el uso 
de ciertas estructuras y colocaciones que muestran un uso especialmente significativo en nuestro corpus y que, por tanto, pueden ser representativas del discurso turístico en el género promocional.

Por último, hemos tratado de determinar si el uso de la adjetivación, tanto a nivel cualitativo como cuantitativo, está ligado con el género web promocional, es decir, si en el género promocional web se da, como cabe esperar, un uso elevado y a veces un tanto hiperbólico de la adjetivación.

\section{Resultados del análisis}

Como ya hemos dicho, la primera parte de los resultados obtenidos con WST y de la cual hemos intentado extraer conclusiones es la relativa a los listados de frecuencia. Estos listados permiten observar y analizar las categorías más comunes en los lenguajes de especialidad y aunque, como apunta Vargas Sierra (2005), las unidades léxicas del lenguaje se materializan en todas las categorías posibles, presentan siempre lo que se podrían denominar "categorías preferentes". La categoría gramatical más común en la cual se materializan los términos es, con mucha diferencia, el nombre, seguido del adjetivo y del verbo, si bien esta última categoría se da en una proporción mucho menor. En este sentido, el marcado carácter nominal de los lenguajes de especialidad parece estar fuera de toda duda (Sager et al. 1980; Cabré Castellvi 1993; Lerat 1995 entre muchos otros). Si observamos las Tablas 3 y 4 apreciaremos cuáles son las 10 unidades léxicas más frecuentes en cada lengua de nuestro corpus de estudio, lo cual nos permitirá además concluir a priori y a grandes rasgos si dicho corpus ha estado correctamente compilado dependiendo de si las palabras más frecuentes están estrechamente relacionadas con la promoción del destino turístico y de su alojamiento, y son lo que podríamos llamar términos o unidades léxicas "esperables" dada la temática de estudio. Hay que apuntar, no obstante, que en ambos casos, tanto para el corpus en inglés como en español, se ha llevado previamente a cabo un filtrado de palabras gramaticales. Es decir, para el análisis de resultados significativos es necesario hacer una preselección de las que realmente son las palabras más frecuentes en cualquier corpus, esto es, las palabras gramaticales o funcionales: una serie de palabras vacías de contenido y sin un valor específico para los recuentos de frecuencia o para fines terminológicos/terminográficos pero extremadamente comunes y frecuentes en cualquier tipo de texto (artículos, preposiciones, etc.). Una vez eliminadas estas palabras de nuestro corpus mediante la aplicación de listados a modo de filtro en la aplicación Wordlist, tanto en español como en inglés, observamos que 9 de las 10 palabras más frecuentes en nuestro corpus son nombres y que la unidad restante es en ambos casos un adjetivo (avai- 
lable y gran). Además, la unidad léxica más común es, en ambos casos y con mucha diferencia, hotel, lo cual no es de extrañar, puesto que la parte más amplia (con mayor número de palabras) en la composición de ambos corpus es la correspondiente a la promoción de alojamientos turísticos (hoteles) en los diversos destinos. Deducimos que por la misma razón las palabras servicio y service/services también aparecen en ambos listados con frecuencias muy elevadas y lo mismo sucede con otras unidades léxicas estrechamente relacionadas con la gestión hotelera: breakfast, available, family o habitaciones. No sorprende tampoco encontrar entre las más frecuentes en inglés la palabra London, ya que es uno de los destinos turísticos sobre los que más información se ha vertido en el corpus. Lo mismo sucede, pero a nivel general y en español, con la unidad léxica ciudad (dado que la mayoría de los destinos turísticos promocionados son ciudades) o con otras como centro o zona. Observando pues ambos listados y la composición del corpus creemos que parece haber una coherencia interna entre el tema objeto de análisis y el corpus de estudio, lo cual es un requisito fundamental antes de proceder a cualquier análisis más detallado.

Puesto que el centro de nuestra investigación son los adjetivos, diremos que, tal y como se muestra en la Tabla 5, de las 1000 primeras unidades léxicas más frecuentes en inglés, 213 son adjetivos. Esto implica que un $21,3 \%$ de las 1000

TABLA 3

Las 10 unidades léxicas más frecuentes del corpus INGLÉS

\begin{tabular}{|c|l|c|}
\hline $\begin{array}{c}\text { Orden según } \\
\text { frecuencia }\end{array}$ & Unidad léxica & $\begin{array}{c}\text { Frecuencia en el corpus } \\
\text { INGLÉS (n }{ }^{0} \text { apariciones) }\end{array}$ \\
\hline 1 & HOTEL & 1241 \\
\hline 2 & LONDON & 951 \\
\hline 3 & FACILITIES & 554 \\
\hline 4 & GUESTS & 478 \\
\hline 5 & SERVICES & 477 \\
\hline 6 & BREAKFAST & 435 \\
\hline 7 & AVAILABLE & 431 \\
\hline 8 & SERVICE & 378 \\
\hline 9 & FAMILY & 369 \\
\hline 10 & INFORMATION & 366 \\
\hline
\end{tabular}


TABLA 4

Las 10 unidades léxicas más frecuentes del corpus ESPAÑOL

\begin{tabular}{|c|l|c|}
\hline $\begin{array}{c}\text { Orden según } \\
\text { frecuencia }\end{array}$ & Unidad léxica & $\begin{array}{c}\text { Frecuencia en el corpus } \\
\text { ESPAÑOL ( }{ }^{0} \text { apariciones) }\end{array}$ \\
\hline 1 & HOTEL & 1018 \\
\hline 2 & HABITACIONES & 572 \\
\hline 3 & CIUDAD & 442 \\
\hline 4 & SERVICIOS & 332 \\
\hline 5 & RESTAURANTE & 296 \\
\hline 6 & CENTRO & 295 \\
\hline 7 & ZONA & 278 \\
\hline 8 & ENCUENTRA & 272 \\
\hline 9 & SERVICIO & 272 \\
\hline 10 & GRAN & 258 \\
\hline
\end{tabular}

palabras más frecuentes en inglés son adjetivos, es decir, aproximadamente una quinta parte. Del mismo modo, tal y como podemos observar en la Tabla 6, de las 1000 primeras unidades léxicas más frecuentes en español, 198 son adjetivos; o sea que un $19,8 \%$ de las 1000 palabras más frecuentes en español son adjetivos, lo que también equivale aproximadamente a una quinta parte de las unidades léxicas analizadas. Bajo el punto de vista cuantitativo, el número de adjetivos en ambas lenguas parece similar y equilibrado sin que pueda afirmarse que ninguna de las dos haga un uso significativamente mayor de la adjetivación.

Somos conscientes de que muchas de las palabras aquí contenidas pueden pertenecer a otras categorias gramaticales además de al adjetivo pero se ha decidido incluirlas bajo el epígrafe único de "adjetivo" por cuestiones prácticas y operativas, si bien debe tenerse en cuenta que las frecuencias de algunas de estas unidades léxicas con varias categorías posibles y consideradas aquí como adjetivos exclusivamente no son reales, porque computan la frecuencia de la palabra en cualquiera de sus categorías.

También hay que apuntar que las listas no han sido lematizadas, por lo que, con fines comparativos, ejemplos como double (291), deberían computar en español como 149 ocurrencias totales, resultantes de sumar los lexemas doble (88) y dobles (61). 
TABLA 5

Adjetivos en inglés y frecuencia de los mismos (seleccionados de entre las $\mathbf{1 0 0 0}$ unidades léxicas más frecuentes en el corpus)

\begin{tabular}{|c|c|c|}
\hline $\begin{array}{c}\text { Orden en } \\
\text { el listado } \\
\text { de frec. }\end{array}$ & Adjetivo & Frec. \\
\hline 7 & AVAILABLE & 431 \\
\hline 18 & DOUBLE & 291 \\
\hline 28 & FREE & 253 \\
\hline 42 & PRIVATE & 216 \\
\hline 44 & STANDARD & 203 \\
\hline 51 & LUXURY & 187 \\
\hline 57 & SPECIAL & 177 \\
\hline 69 & PERSONAL & 159 \\
\hline 76 & SINGLE & 153 \\
\hline 86 & PERFECT & 145 \\
\hline 90 & COMFORTABLE & 142 \\
\hline 92 & CENTRAL & 141 \\
\hline 95 & IDEAL & 138 \\
\hline 96 & FRIENDLY & 137 \\
\hline 101 & TWIN & 135 \\
\hline 108 & MODERN & 126 \\
\hline 119 & DIRECT & 116 \\
\hline 120 & ONLINE & 116 \\
\hline 130 & SELF & 110 \\
\hline 133 & PUBLIC & 108 \\
\hline 136 & SHORT & 107 \\
\hline 155 & BEAUTIFUL & 91 \\
\hline
\end{tabular}

\begin{tabular}{|c|c|c|}
\hline $\begin{array}{c}\text { Orden en } \\
\text { el listado } \\
\text { de frec. }\end{array}$ & Adjetivo & Frec. \\
\hline 158 & LOCAL & 89 \\
\hline 175 & SPACIOUS & 83 \\
\hline 179 & COMPLETE & 82 \\
\hline 181 & REQUIRED & 82 \\
\hline 189 & EASY & 79 \\
\hline 193 & EXCELLENT & 78 \\
\hline 220 & LEISURE & 71 \\
\hline 235 & MINIMUM & 67 \\
\hline 252 & LUXURIOUS & 64 \\
\hline 255 & TRADITIONAL & 64 \\
\hline 266 & ACCEPTED & 61 \\
\hline 269 & DESIGNED & 61 \\
\hline 275 & BRITISH & 60 \\
\hline 281 & TRIPLE & 59 \\
\hline 286 & LIMITED & 58 \\
\hline 294 & CONTINENTAL & 56 \\
\hline 300 & CONTEMPORARY & 55 \\
\hline 310 & FOLLOWING & 54 \\
\hline 315 & UNITED & 54 \\
\hline 320 & MAJOR & 53 \\
\hline 335 & INTERNATIONAL & 51 \\
\hline 341 & WIRELESS & 51 \\
\hline
\end{tabular}


TABLA 5 (Cont.)

\begin{tabular}{|c|c|c|}
\hline $\begin{array}{l}\text { Orden en } \\
\text { el listado } \\
\text { de frec. }\end{array}$ & Adjetivo & Frec. \\
\hline 358 & CORPORATE & 48 \\
\hline 362 & LARGEST & 48 \\
\hline 364 & SHARING & 48 \\
\hline 365 & SQUARE & 48 \\
\hline 368 & COMMITTED & 47 \\
\hline 371 & GRAND & 47 \\
\hline 376 & SAFE & 47 \\
\hline 383 & NEAREST & 46 \\
\hline 385 & SUPERIOR & 46 \\
\hline 392 & RELAXING & 45 \\
\hline 395 & ADDITIONAL & 44 \\
\hline 399 & EXTRA & 44 \\
\hline 401 & INCLUDED & 44 \\
\hline 408 & FURNISHED & 43 \\
\hline 410 & HISTORIC & 43 \\
\hline 411 & STYLISH & 43 \\
\hline 421 & BUDGET & 41 \\
\hline 431 & DECORATED & 40 \\
\hline 435 & NATURAL & 40 \\
\hline 438 & SEASONAL & 40 \\
\hline 447 & LITTLE & 39 \\
\hline 450 & NATIONAL & 39 \\
\hline 456 & ACCESSIBLE & 38 \\
\hline 469 & WONDERFUL & 38 \\
\hline
\end{tabular}

\begin{tabular}{|c|l|c|}
\hline $\begin{array}{c}\text { Orden en } \\
\text { el listado } \\
\text { de frec. }\end{array}$ & Adjetivo & Frec. \\
\hline 470 & COSY & 37 \\
\hline 472 & DRY & 37 \\
\hline 478 & PRIOR & 37 \\
\hline 480 & RELATED & 37 \\
\hline 484 & ELEGANT & 36 \\
\hline 487 & EXECUTIVE & 36 \\
\hline 488 & FRESH & 36 \\
\hline 494 & WHITE & 36 \\
\hline 497 & CLASSIC & 35 \\
\hline 498 & COMPLEX & 35 \\
\hline 499 & EXPRESS & 35 \\
\hline 503 & SITTING & 35 \\
\hline 512 & FINE & 34 \\
\hline 516 & LOWER & 34 \\
\hline 523 & VALID & 34 \\
\hline 528 & FAMOUS & 33 \\
\hline 536 & UNIQUE & 33 \\
\hline 543 & FANTASTIC & 32 \\
\hline 555 & SHARED & 32 \\
\hline 559 & WELCOMING & 32 \\
\hline 567 & LATE & 31 \\
\hline 572 & SECURE & 31 \\
\hline 578 & ADDED & 30 \\
\hline 582 & EXTERNAL & 30 \\
\hline
\end{tabular}


TABLA 5 (Cont.)

\begin{tabular}{|c|c|c|c|c|c|}
\hline $\begin{array}{l}\text { Orden en } \\
\text { el listado } \\
\text { de frec. }\end{array}$ & Adjetivo & Frec. & $\begin{array}{c}\text { Orden en } \\
\text { el listado } \\
\text { de frec. }\end{array}$ & Adjetivo & Frec. \\
\hline 587 & LEGAL & 30 & 690 & OUTSTANDING & 25 \\
\hline 588 & LOVELY & 30 & 696 & VEGETARIAN & 25 \\
\hline 591 & PROFESSIONAL & 30 & 698 & ACTIVE & 24 \\
\hline 593 & REAL & 30 & 700 & APPLICABLE & 24 \\
\hline 597 & EXTENSIVE & 29 & 739 & LICENSED & 23 \\
\hline 600 & PEACEFUL & 29 & 745 & ROUND & 23 \\
\hline 602 & RELAXED & 29 & 749 & SPECTACULAR & 23 \\
\hline 609 & CHARMING & 28 & 758 & ALLOWED & 22 \\
\hline 618 & OPTIONAL & 28 & 770 & HAPPY & 22 \\
\hline 620 & POPULAR & 28 & 772 & IMPRESSIVE & 22 \\
\hline 622 & ROMANTIC & 28 & 774 & PREVIOUS & 22 \\
\hline 623 & ROYAL & 28 & 789 & COMBINED & 21 \\
\hline 648 & TOURIST & 27 & 790 & CONVENIENT & 21 \\
\hline 661 & CONSIDERED & 26 & 791 & DEDICATED & 21 \\
\hline 663 & DELIGHTFUL & 26 & 792 & DELICIOUS & 21 \\
\hline 666 & EXCLUSIVE & 26 & 795 & EXCEPTIONAL & 21 \\
\hline 667 & FABULOUS & 26 & 797 & FLEXIBLE & 21 \\
\hline 668 & FINEST & 26 & 801 & INTERACTIVE & 21 \\
\hline 673 & ORIGINAL & 26 & 819 & APPROPRIATE & 20 \\
\hline 674 & OUTDOOR & 26 & 825 & BUSY & 20 \\
\hline 679 & SPECIFIC & 26 & 849 & PICTURESQUE & 20 \\
\hline 680 & STUNNING & 26 & 852 & REFURBISHED & 20 \\
\hline 681 & SUPERB & 26 & 853 & RESPONSIBLE & 20 \\
\hline 686 & HOT & 25 & 856 & SUITABLE & 20 \\
\hline
\end{tabular}




\section{TABLA 5 (Cont.)}

\begin{tabular}{|c|l|c|}
\hline $\begin{array}{c}\text { Orden en } \\
\text { el listado } \\
\text { de frec. }\end{array}$ & \multicolumn{1}{|c|}{ Adjetivo } & Frec. \\
\hline 857 & SUPER & 20 \\
\hline 860 & WORLDWIDE & 20 \\
\hline 864 & AMERICAN & 19 \\
\hline 867 & AWARE & 19 \\
\hline 875 & CONDITIONED & 19 \\
\hline 882 & EXCITING & 19 \\
\hline 891 & LISTED & 19 \\
\hline 893 & MAGNIFICENT & 19 \\
\hline 896 & MEMORABLE & 19 \\
\hline 910 & STRONG & 19 \\
\hline 918 & VARIOUS & 19 \\
\hline 923 & BLACK & 18 \\
\hline 926 & CHARGED & 18 \\
\hline 931 & DESIGNATED & 18 \\
\hline 932 & DESIRABLE & 18 \\
\hline 935 & EQUAL & 18 \\
\hline
\end{tabular}

\begin{tabular}{|c|l|c|}
\hline $\begin{array}{c}\text { Orden en } \\
\text { el listado } \\
\text { de frec. }\end{array}$ & Adjetivo & Frec. \\
\hline 939 & FITTED & 18 \\
\hline 946 & LOW & 18 \\
\hline 948 & MID & 18 \\
\hline 951 & ORGANIC & 18 \\
\hline 959 & REGARDING & 18 \\
\hline 960 & REGULAR & 18 \\
\hline 964 & SELECTED & 18 \\
\hline 965 & SEND & 18 \\
\hline 966 & SERVICED & 18 \\
\hline 983 & COMPREHENSIVE & 17 \\
\hline 985 & CONVERTED & 17 \\
\hline 987 & DELIVERED & 17 \\
\hline 988 & DENTAL & 17 \\
\hline 990 & DISABLED & 17 \\
\hline 994 & ENJOYABLE & 17 \\
\hline 998 & INDOOR & 17 \\
\hline
\end{tabular}

TABLA 6

Adjetivos en español y frecuencia de los mismos (seleccionados de entre las $\mathbf{1 0 0 0}$ unidades léxicas más frecuentes en el corpus)

\begin{tabular}{|c|l|c|}
\hline $\begin{array}{c}\text { Orden en } \\
\text { el listado } \\
\text { de frec. }\end{array}$ & \multicolumn{1}{|c|}{ Adjetivo } & Frec. \\
\hline 10 & GRAN & 258 \\
\hline 51 & NATURAL & 136 \\
\hline 60 & SANTA & 125 \\
\hline
\end{tabular}

\begin{tabular}{|c|l|c|}
\hline $\begin{array}{c}\text { Orden en } \\
\text { el listado } \\
\text { de frec. }\end{array}$ & \multicolumn{1}{|c|}{ Adjetivo } & Frec. \\
\hline 68 & ACONDICIONADO & 115 \\
\hline 72 & INTERIOR & 111 \\
\hline 73 & MAYOR & 111 \\
\hline
\end{tabular}


TABLA 6 (Cont.)

\begin{tabular}{|c|c|c|}
\hline $\begin{array}{l}\text { Orden en } \\
\text { el listado } \\
\text { de frec. }\end{array}$ & Adjetivo & Frec. \\
\hline 85 & CERCA & 97 \\
\hline 88 & REAL & 96 \\
\hline 93 & MEJOR & 94 \\
\hline 102 & ANTIGUO & 90 \\
\hline 105 & IDEAL & 89 \\
\hline 107 & DOBLE & 88 \\
\hline 115 & TRADICIONAL & 85 \\
\hline 119 & COMPLETO & 82 \\
\hline 122 & GRANDES & 81 \\
\hline 129 & RURAL & 79 \\
\hline 130 & UBICADO & 79 \\
\hline 133 & EQUIPADAS & 77 \\
\hline 136 & MODERNO & 76 \\
\hline 137 & ANTIGUA & 75 \\
\hline 138 & CULTURAL & 75 \\
\hline 144 & PRIMERA & 73 \\
\hline 153 & MEJORES & 71 \\
\hline 155 & FUERTE & 70 \\
\hline 156 & NATURALES & 70 \\
\hline 177 & CENTRAL & 66 \\
\hline 179 & LIBRE & 65 \\
\hline 183 & ESPECIAL & 64 \\
\hline 184 & PRINCIPAL & 64 \\
\hline 193 & DOBLES & 61 \\
\hline
\end{tabular}

\begin{tabular}{|c|c|c|}
\hline $\begin{array}{l}\text { Orden en } \\
\text { el listado } \\
\text { de frec. }\end{array}$ & Adjetivo & Frec. \\
\hline 194 & EXTERIOR & 61 \\
\hline 195 & IMPORTANTE & 61 \\
\hline 197 & NUEVO & 61 \\
\hline 210 & ELEGANTE & 58 \\
\hline 215 & BUEN & 57 \\
\hline 223 & TURISTICO & 57 \\
\hline 233 & AMPLIA & 55 \\
\hline 241 & TRADICIONALES & 54 \\
\hline 248 & GRATUITO & 52 \\
\hline 249 & IMPORTANTES & 52 \\
\hline 255 & TURISTICA & 52 \\
\hline 256 & DIFERENTES & 51 \\
\hline 258 & INTERNACIONAL & 51 \\
\hline 260 & ALTA & 50 \\
\hline 272 & NACIONAL & 49 \\
\hline 276 & ÁRABE & 48 \\
\hline 282 & MEDIO & 48 \\
\hline 284 & PROPIO & 48 \\
\hline 293 & PRINCIPALES & 46 \\
\hline 295 & ÁRABES & 45 \\
\hline 296 & BUENA & 45 \\
\hline 299 & EXCELENTE & 45 \\
\hline 307 & INDIVIDUALES & 44 \\
\hline 309 & NUMEROSOS & 44 \\
\hline
\end{tabular}


TABLA 6 (Cont.)

\begin{tabular}{|c|l|c|}
\hline $\begin{array}{c}\text { Orden en } \\
\text { el listado } \\
\text { de frec. }\end{array}$ & Adjetivo & Frec. \\
\hline 310 & PRIVADO & 44 \\
\hline 316 & SITUADA & 43 \\
\hline 317 & AMPLIAS & 42 \\
\hline 320 & GÓTICO & 42 \\
\hline 323 & PEQUEÑO & 42 \\
\hline 333 & GENERAL & 41 \\
\hline 334 & MODERNAS & 41 \\
\hline 335 & NUMEROSAS & 41 \\
\hline 336 & PRIMER & 41 \\
\hline 345 & PERFECTO & 40 \\
\hline 350 & TOTAL & 40 \\
\hline 353 & CONOCIDA & 39 \\
\hline 355 & DECORADAS & 39 \\
\hline 361 & ACOGEDOR & 38 \\
\hline 362 & AGRADABLE & 38 \\
\hline 371 & ÚLTIMO & 38 \\
\hline 382 & NUEVA & 37 \\
\hline 390 & FÁCIL & 36 \\
\hline 392 & LOCAL & 36 \\
\hline 399 & RODEADO & 36 \\
\hline 401 & SINGULAR & 36 \\
\hline 403 & ÚLTIMA & 36 \\
\hline 409 & CONFORTABLE & 35 \\
\hline 411 & DISPONIBLE & 35 \\
\hline
\end{tabular}

\begin{tabular}{|c|c|c|}
\hline $\begin{array}{l}\text { Orden en } \\
\text { el listado } \\
\text { de frec. }\end{array}$ & Adjetivo & Frec. \\
\hline 414 & FAMOSA & 35 \\
\hline 426 & ALTO & 34 \\
\hline 427 & ARTÍSTICO & 34 \\
\hline 433 & FINALES & 34 \\
\hline 435 & INDIVIDUAL & 34 \\
\hline 438 & MÁXIMO & 34 \\
\hline 446 & BAJA & 33 \\
\hline 455 & SUPLETORIA & 33 \\
\hline 460 & CLÁSICO & 32 \\
\hline 462 & ELEGANTES & 32 \\
\hline 463 & ESPECIALES & 32 \\
\hline 468 & SIGUIENTES & 32 \\
\hline 471 & URBANO & 32 \\
\hline 474 & CONFORTABLES & 31 \\
\hline 479 & PEQUEÑOS & 31 \\
\hline 480 & POPULAR & 31 \\
\hline 484 & SANTO & 31 \\
\hline 487 & ÚNICO & 31 \\
\hline 491 & DIFERENTE & -30 \\
\hline 493 & ESPECTACULAR & 30 \\
\hline 496 & LITORAL & 30 \\
\hline 498 & MEDIEVAL & 30 \\
\hline 499 & MEDITERRÁNEO & 30 \\
\hline 500 & MODERNA & 30 \\
\hline
\end{tabular}


TABLA 6 (Cont.)

\begin{tabular}{|c|c|c|}
\hline $\begin{array}{c}\text { Orden en } \\
\text { el listado } \\
\text { de frec. }\end{array}$ & Adjetivo & Frec. \\
\hline 511 & CONTEMPORÁNEO & 29 \\
\hline 520 & ORIGINAL & 29 \\
\hline 522 & POPULARES & 29 \\
\hline 523 & PRIVILEGIADA & 29 \\
\hline 528 & ACTUAL & 28 \\
\hline 533 & FAMOSO & 28 \\
\hline 536 & JUNIOR & 28 \\
\hline 537 & JUSTO & 28 \\
\hline 544 & ÚNICA & 28 \\
\hline 547 & AMPLIO & 27 \\
\hline 553 & LUJOSO & 27 \\
\hline 558 & MONUMENTAL & 27 \\
\hline 561 & PEQUEÑA & 27 \\
\hline 564 & TIPICA & 27 \\
\hline 566 & ABIERTA & 26 \\
\hline 570 & CERCANO & 26 \\
\hline 582 & GRANADINO & 26 \\
\hline 585 & INAUGURADO & 26 \\
\hline 599 & SUPERIOR & 26 \\
\hline 605 & ANTIGUAS & 25 \\
\hline 607 & CALIENTE & 25 \\
\hline 610 & FANTÁSTICO & 25 \\
\hline 611 & EQUIPADA & 25 \\
\hline 612 & ESPECTACULARES & 25 \\
\hline
\end{tabular}

\begin{tabular}{|c|c|c|}
\hline $\begin{array}{c}\text { Orden en } \\
\text { el listado } \\
\text { de frec. }\end{array}$ & Adjetivo & Frec. \\
\hline 613 & FERIAL & 25 \\
\hline 614 & GRANDE & 25 \\
\hline 618 & MAYORES & 25 \\
\hline 631 & ANDALUZ & 24 \\
\hline 633 & BELLAS & 24 \\
\hline 648 & PRÓXIMO & 24 \\
\hline 649 & PÚBLICO & 24 \\
\hline 655 & TRANQUILO & 24 \\
\hline 656 & TURISTICOS & 24 \\
\hline 658 & ACTIVO & 23 \\
\hline 661 & ANTIGUOS & 23 \\
\hline 664 & BLANCO & 23 \\
\hline 666 & COMPLETA & 23 \\
\hline 667 & COMUNES & 23 \\
\hline 669 & CONVERTIDO & 23 \\
\hline 671 & CUADRADOS & 23 \\
\hline 673 & ESCASOS & 23 \\
\hline 674 & EXCLUSIVO & 23 \\
\hline 682 & ORIGINALES & -23 \\
\hline 683 & PALACIOS & 23 \\
\hline 703 & ESPAÑOLA & 22 \\
\hline 704 & ESTÁNDAR & 22 \\
\hline 709 & INTERESANTE & 22 \\
\hline 712 & NOBLES & 22 \\
\hline
\end{tabular}


TABLA 6 (Cont.)

\begin{tabular}{|c|l|c|}
\hline $\begin{array}{c}\text { Orden en } \\
\text { el listado } \\
\text { de frec. }\end{array}$ & Adjetivo & Frec. \\
\hline 715 & PLENA & 22 \\
\hline 717 & POSIBLE & 22 \\
\hline 740 & EXCELENTES & 21 \\
\hline 746 & HISTÓRICOS & 21 \\
\hline 753 & PERFECTA & 21 \\
\hline 756 & RENACENTISTA & 21 \\
\hline 772 & BARROCA & 20 \\
\hline 773 & BARROCO & 20 \\
\hline 779 & CONOCIDO & 20 \\
\hline 780 & CUIDADO & 20 \\
\hline 782 & DISPONIBLES & 20 \\
\hline 789 & HISTÓRICA & 20 \\
\hline 792 & IMPRESIONANTE & 20 \\
\hline 794 & MAGNIFICO & 20 \\
\hline 805 & PRÁCTICA & 20 \\
\hline 808 & REALES & 20 \\
\hline 813 & SEGUNDA & 20 \\
\hline 815 & TRANQUILA & 20 \\
\hline 826 & CULTURALES & 19 \\
\hline 836 & GASTRONÓMICA & 19 \\
\hline 839 & HERMOSOS & 19 \\
\hline 846 & NECESARIO & 19 \\
\hline 850 & PRIMEROS & 19 \\
\hline 861 & ÚLTIMAS & 19 \\
\hline
\end{tabular}

\begin{tabular}{|c|l|c|}
\hline $\begin{array}{c}\text { Orden en } \\
\text { el listado } \\
\text { de frec. }\end{array}$ & \multicolumn{1}{|c|}{ Adjetivo } & Frec. \\
\hline 864 & ALTAS & 18 \\
\hline 869 & ARTESANAL & 18 \\
\hline 872 & BONITO & 18 \\
\hline 882 & ELABORADOS & 18 \\
\hline 884 & ESPACIOSAS & 18 \\
\hline 908 & RELAJANTE & 18 \\
\hline 910 & ROMÁNTICA & 18 \\
\hline 916 & ÚLTIMOS & 18 \\
\hline 919 & VIEJA & 18 \\
\hline 937 & DEPORTIVAS & 17 \\
\hline 940 & DISTINTAS & 17 \\
\hline 942 & EXCEPCIONAL & 17 \\
\hline 943 & EXQUISITA & 17 \\
\hline 944 & EXQUISITOS & 17 \\
\hline 945 & EXTREMO & 17 \\
\hline 946 & FAMILIAR & 17 \\
\hline 951 & INTERESANTES & 17 \\
\hline 954 & MAGNIFICA & 17 \\
\hline 961 & PEQUEÑAS & -17 \\
\hline 965 & RURALES & 17 \\
\hline 967 & SITUADOS & 17 \\
\hline 972 & VARIADA & 17 \\
\hline 976 & ANDALUZA & 16 \\
\hline 995 & CUIDADA & 16 \\
\hline
\end{tabular}


Como apuntábamos entre los objetivos de este estudio, vamos a proceder a clasificar los adjetivos más relevantes de las Tablas 5 y 6 de manera que podamos establecer una tipología de los adjetivos más comunes o más utilizados en el género web promocional. Para ello, partiremos de la tipología de Pierini (2009), adaptándola a lo que creemos que es nuestro contexto de estudio y nuestras necesidades. Así pues, basándonos en la observación de los adjetivos obtenidos en ambas lenguas de estudio y en la clasificación de Pierini (2009), podemos establecer que las principales categorías en las que podríamos englobar los adjetivos extraidos de nuestro corpus serian:

\section{TABLA 7}

\section{Propuesta de tipología de adjetivos para este estudio}

1. Adjetivos descriptivo-verificables (referidos a características fisicas, objetivas y verificables del tipo espacio/tamaño, cantidad).

2. Adjetivos objetivo-intangibles (referidos a características objetivas pero intangibles del tipo tiempo, disponibilidad, coste).

3. Adjetivos de singularidad, originalidad $y / 0$ exclusividad.

4. Adjetivos estético-sensoriales.

5. Adjetivos de bienestar o comodidad.

6. Adjetivos "excelsos" (referidos a lo extraordinario).

7. Adjetivos de popularidad.

8. Adjetivos de exaltación de lo propio (autenticidad).

9. Adjetivos de calado emocional.

10. Adjetivos de modernidad-tradición.

Hemos intentado a continuación ilustrar la tipología arriba mostrada con adjetivos extraídos de nuestro corpus de estudio para ver si dicha tipología se adecúa a las categorías más utilizadas en el género web promocional de destinos turísticos. Hemos incluido la frecuencia con que dichos adjetivos aparecen en el corpus de manera que la clasificación de los adjetivos represente de forma más fiable la representatividad de los mismos en el dominio, si bien todos ellos pueden ser considerados significativos a priori porque figuran entre las 1000 palabras más frecuentes de nuestro corpus. 
1. Adjetivos descriptivo-verificables (referidos a características físicas, objetivas y verificables del tipo espacio/tamaño, cantidad).

Dobles (61), individuales (44), gótico (42), pequeño (42), decoradas (39), individual (34), supletoria (33), medieval (30), blanco (23), estándar (22), renacentista $(21)$, barroca $(20) \ldots$

Single (153), twin (135), spacious (83), triple (59), wireless (51), white (36), refurbished (20), black (18)...

2. Adjetivos objetivo-intangibles (referidos a características objetivas pero intangibles del tipo tiempo, disponibilidad, coste).

Interior (111), cultural (75), exterior (61), gratuito (52), internacional (51), nacional (49), privado (44), disponible (35)...

Available (431), standard (203), international (51), historic (43), budget (41), executive (36), optional (28), licensed (23), serviced (18), indoor (17)...

3. Adjetivos de singularidad, originalidad y/o exclusividad.

Singular (36), único (31), diferente (30), original (29), exclusivo (23), única (28), exclusivo (23), originales (23)...

Unique (33), original (26), exclusive (26), exceptional (21)...

\section{Adjetivos estético-sensoriales.}

Entre los adjetivos que pretenden llegar al turista potencial a través de la evocación sensorial y de la exaltación de las cualidades estéticas de un destino encontramos:

Especial (64), elegante (58), privilegiada (29), lujoso (27), espectaculares (25), bellas (24), activo (23), originales (23), impresionante (20), tranquila (20), hermosos (19), relajante (18), bonito (18), exquisita (17), exquisi$\operatorname{tos}(17) \ldots$

Luxury (187), beautiful (91), luxurious (64), relaxing (45), stylish (43), elegant (36), fantastic (32), peaceful (29), delightful (26), fabulous (26), stunning (26), happy (22), delicious (21), picturesque (20)... 


\section{Adjetivos de bienestar o comodidad.}

Privado (44), acogedor (38), agradable (38), confortable (35), confortables (31), accesible (38)...

Private (216), comfortable (142), cosy (37), welcoming (32), suitable (20)...

\section{Adjetivos "excelsos" (referidos a lo extraordinario).}

Entre los adjetivos que ensalzan de manera superlativa las bondades de un destino, hallamos:

Mejor (94), ideal (89), mejores (71), principal (64), excelente (45), perfecto (40), máximo (34), privilegiada (29), superior (26), excelentes (21), perfecta (21), magnifico (20), excepcional (17), extremo (17), magnífica (17)...

Perfect (145), ideal (138), excellent (78), major (53), largest (48), grand (47), superior (46), wonderful (38), delightful (26), fabulous (26), finest (26), superb (26), outstanding (26), spectacular (23), impressive (22), super (20), magnificent (19)...

\section{Adjetivos de popularidad.}

Famosa (35), popular (31), populares (29), famoso (28)...

Famous (33), popular (28)...

\section{Adjetivos de exaltación de lo propio (autenticidad).}

Tradicional (85), árabe (48), árabes (45), local (36), tipica (27), granadino (26), andaluz (24), española (22), artesanal (18), andaluza (16)...

Local (89), British (60), American (19)...

\section{Adjetivos de calado emocional.}

Especial (64), natural (40), agradable (38), romántica (18)...

Natural (136), friendly (130), lovely (30), romantic (28), charming (28), exciting (19), desirable (18), enjoyable (17)... 


\section{Adjetivos de modernidad-tradición.}

Antiguo (90), tradicional (85), moderno (76), antigua (75), modernas (41), conocida (39), nueva (37), moderna (30), contemporáneo (29), actual (28), antiguas (25), antiguos (23), histórica (20), conocido (20), vieja $(18) \ldots$

Modern (126), contemporary (55), classic (35)...

Apreciamos un predominio de los adjetivos descriptivo-verificables -que atribuimos a la necesidad de proporcionar información real, objetiva, funcional y útil en el sentido estricto de la palabra-, cosa que también ocurre con los adjetivos objetivo-intangibles. Del mismo modo los adjetivos estético-sensoriales y excelsos juegan un papel fundamental y, por otra parte, nada sorprendente, puesto que corroboran la importancia de atraer al turista por los sentidos ensalzando las bondades de los destinos de forma muy marcada.

Además, entre los adjetivos mostrados en las Tablas 5 y 6 , apreciamos la gran cantidad de lexemas que coinciden en ambas tablas como significativos, es decir que son muchos los conceptos representados a través de adjetivos que se dan tanto entre los adjetivos más comunes en inglés como en español en nuestro corpus. La Tabla 8 muestra parte de estas equivalencias comunes entre los adjetivos más frecuentes en ambos corpus. De esto podemos deducir que los adjetivos mediante los que se pretende promocionar un destino suelen ser muy similares en la mayoría de los casos en ambas lenguas y suelen, por tanto, hacer referencia al mismo tipo de cualidades y estados e incidir en los mismos aspectos, por lo que tanto el inglés como el español harían un uso de la adjetivación similar en las páginas web promocionales de los destinos turísticos.

Por último, observamos que tanto en inglés como en español es común y esperable el uso de comparativos y superlativos a la hora de definir las cualidades de los destinos turísticos. Para analizar este uso, que hemos detectado como frecuente en el género web promocional turístico, hemos centrado nuestra búsqueda -con la aplicación Collocates de Concord- en las palabras más y more (en el caso de los comparativos en ambas lenguas) y en el/la/los/las más, the most y the ${ }^{*}$ est $t^{1}$ (en el caso de los superlativos en ambos idiomas). Hemos detectado por este medio que es algo más común el uso de las estructuras comparativas con

1 The * est es el lenguaje de búsqueda utilizado para indicarle a WST que queremos recuperar todas aquellas palabras que acaban en "est" y que tienen delante un the, puesto que esta es la manera en que se forma el superlativo de los adjetivos "largos" en inglés. 
TABLA 8

Equivalencias comunes entre los adjetivos más frecuentes en español e inglés en nuestro corpus

\begin{tabular}{|c|c|c|}
\hline available-disponible & $\begin{array}{c}\text { comfortable - confortable } \\
\text { confortables }\end{array}$ & spacious - espaciosas \\
\hline double - doble, dobles & central-central & $\begin{array}{c}\text { complete - completo, } \\
\text { completa }\end{array}$ \\
\hline free-gratuito & ideal-ideal & easy-fácil \\
\hline private-privado & friendly - agradable & $\begin{array}{l}\text { excellent-excelente, } \\
\text { excelentes }\end{array}$ \\
\hline luxury, luxurious-lujoso & $\begin{array}{l}\text { modern- moderno, } \\
\text { moderna, modernas }\end{array}$ & $\begin{array}{c}\text { traditional-tradicional, } \\
\text { tradicionales }\end{array}$ \\
\hline $\begin{array}{l}\text { special-especial, } \\
\text { especiales }\end{array}$ & public-público & $\begin{array}{l}\text { contemporary - } \\
\text { contemporáneo }\end{array}$ \\
\hline $\begin{array}{l}\text { single - individual, } \\
\text { individuales }\end{array}$ & $\begin{array}{l}\text { beautiful-bonito, } \\
\text { hermosos, bellas }\end{array}$ & $\begin{array}{c}\text { international - } \\
\text { internacional }\end{array}$ \\
\hline $\begin{array}{l}\text { perfect }- \text { perfecto, } \\
\text { perfecta }\end{array}$ & local-local & Etc. \\
\hline
\end{tabular}

adjetivos en español que en inglés, donde, sin embargo, predominan más los adjetivos que hemos denominado excelsos (y que por tanto de algún modo ya llevan implícita la marca superlativa) y los superlativos que en español. Con Collocates, hemos recuperado los colocados más frecuentes con respecto a la palabra de búsqueda o nodo ordenándolo por posición e indicando su frecuencia en cada caso. Con esta herramienta hemos compilado, pues, las colocaciones más habituales de estos usos en nuestro corpus, habiéndonos centrado en la observación de los colocados de más, more, the most (palabras de búsqueda o nodos) en posición R1, esto es, los que aparecen como primera palabra a la derecha del nodo, por ser los más comunes y significativos, y hemos indicado su frecuencia en dicha posición con respecto al nodo.

Así pues, en español obtenemos:

El/a/los/las más: importantes (32) // importante (15); tradicionales (6) // tradicional (4); grande (11) // grandes (5); populares (9); altas (9); emblemáticos (9); antigua (7); interesantes (6); pequeños (7); antiguo (4); bellas (4); singulares (4); variados (3); agradable (5); concurridas (5); exigentes (5); famosa (4). 
Mientras que en inglés, el programa nos ha recuperado los siguientes colocados como significativos a nivel cuantitativo:

More: enjoyable (4).

The most: discerning (11); most comfortable (9); most important (6).

Del mismo modo, hemos observado la frecuencia de la estructura superlativa inglesa the *est (nodo) -en este caso nos hemos centrado en la frecuencia del nodo en sí y no de los colocados R1- lo que nos ha permitido constatar el uso común de adjetivos superlativos del tipo:

The best (100), the highest (30), the largest (21), the finest (20), the nearest (11), the latest (9) y the richest (7).

Por último, por considerarlo especialmente significativo y característico del género web promocional, tal y como los resultados previos han mostrado, hemos querido corroborar mediante la observación de algunas líneas de concordancia el uso hiperbólico de los adjetivos en este género, planteándonos hasta qué punto la imposibilidad de cumplir con las expectativas generadas en el turista mediante el lenguaje no pueden ser un factor negativo a largo plazo. El "sobreuso" de los adjetivos excelsos o el "sobreuso" de adjetivos de casi cualquier categoría a la hora de ensalzar las bondades de un destino turístico puede ser un ejemplo de lo que Dann (1996) denomina la "técnica de la euforia" (euphoria technique) y que podría ilustrarse con los siguientes ejemplos de nuestro corpus de estudio:

[...] con siete piscinas, la mayor de ellas con un diseño espectacular y vistas sobresalientes, tiene una inteligente distribución que crea zonas semiprivadas.

Situated in a premier position on the castle headland with magnificent sea views of the beautiful North Bay of Scarborough and Yorkshire Coast.

Podemos afirmar sin miedo a equivocarnos y por lo que se desprende de nuestro corpus que esta técnica de la euforia (sean cuales sean sus resultados dependiendo del tipo de turista) se da con asiduidad en el género promocional web en ambas lenguas, lo que hace absolutamente necesario el uso un tanto hiperbólico de los adjetivos.

\section{Conclusiones}

Tal y como se ha visto a lo largo de este estudio, en todo este conglomerado de situaciones, tipologías y aspectos de diversas indole que se conjugan para dar 
lugar, forma y sentido a la industria turística encontramos un género o macrogénero que hoy en día destaca por encima del resto en este sector: la página web. La mercadotecnia del turismo o del destino turístico no se entendería hoy en día sin las páginas web, cuyo componente visual parece innegable y crucial de la misma manera que lo es el lenguaje como transmisor de información concreta y necesaria al turista potencial. Es el lenguaje lo que ha centrado la atención de este estudio y más concretamente el uso de los adjetivos como unidad léxica fundamental a la hora de persuadir, puesto que, como se desprende de la tipología elaborada para su clasificación, los adjetivos pueden expresar significados sensoriales, emocionales, descriptivos..., todos ellos con el fin de transmitir una imagen que atraiga al turista al destino promocionado.

De todo lo visto hasta el momento parece deducirse que los adjetivos contribuyen en gran manera al carácter enfático y evaluativo de la mayoría de páginas web promocionales de destinos turísticos, ya que todas ellas pretenden enfatizar y poner de manifiesto de forma atractiva los aspectos positivos de producto o servicio ofrecido de manera que estos destinos resulten atractivos y conviertan al turista potencial en turista real de nuestro destino.

Obviamente, los resultados obtenidos y, por tanto, el tipo de adjetivos analizados han dependido mucho de la composición del corpus. El hecho de que las páginas web de hoteles de los múltiples destinos turísticos constituyeran un más que importante porcentaje de la composición del corpus no ha hecho sino dar lugar a la aparición de gran cantidad de adjetivos relacionados con el alojamiento y su gestión, que han sido además los adjetivos más específicos de cuantos hemos encontrado $\mathrm{y}$, por consiguiente, los que podríamos calificar como más especializados y también más objetivos por limitarse a transmitir información real y útil del lugar. El resto de adjetivos apelan directamente a la persuasión del turista, siendo especialmente comunes aquellos adjetivos destinados a ensalzar de manera superlativa las cualidades de los destinos turisticos y sus alojamientos, a fomentar una imagen positiva y deseable del destino, presentándolo en muchos casos de manera exagerada. Estos adjetivos destinados a emocionar, a llegar a la subjetividad del receptor, persuadir y, por ende, a promover la acción de viajar al destino elegido son los que predominan en ambas lenguas en el género web promocional del destino turístico.

\section{Referencias bibliográficas}

Álvarez, Charo (1998): "Turismo y nuevas tecnologías", en: Revista Valenciana d'Estudis Autonòmics 25, 135-150, <http://www.pre.gva.es/argos/fileadmin/argos/datos/ RVEA/libro_25/135-25.pdf>. 
BALBONI, Paolo (1989): "La microlingua del turismo come 'fascio di microlingue"”, en: Microlingue e letteratura nella scuola superiore. Brescia: La Scuola, 56-61.

BIGNÉ, J. Enrique/Font, Xavier/ANDREU, Luisa (2000): Marketing de destinos turisticos: análisis y estrategias de desarrollo. Madrid: ESIC.

CABré CAStellvi, María Teresa (1993): La terminologia: teoria, metodologia, aplicaciones. Barcelona: Antártida/Empúries.

CALvı, Maria Vittoria (2010): "Los géneros discursivos en la lengua del turismo: una propuesta de clasificación”, en: Ibérica 19, 9-32.

DanN, Graham (1996): The Language of Tourism: A Sociolinguistic Perspective. Wallingford, CAB International.

EDO MARZA, Nuria (2010): "The discourse community of the tourism industry: a focus on genre conventions for balanced corpus composition", en: Actas del IX Annual Conference of the European Association of Languages for Specific Purposes. Hamburg (Alemania).

LERAT, Pierre (1995): Las lenguas especializadas. Barcelona: Ariel.

MAJó, Joaquim/GALi, Nuria (2002): "Internet en la información turística”, en: IV Congreso Turismo y Tecnologias de la Información y las Comunicaciones. TuriTec, 397-409, $<$ http://www.turismo.uma.es/turitec/turitec2002/actas/Microsoft $\% 20$ Word $\% 20$ \%2026.MAJOGIRONA.pdf>.

PIERINI, Patricia (2009): "Adjectives in tourism English on the web: a corpus-based study”, en: Círculo de Lingüistica Aplicada a la Comunicación 40/2009, 93-116.

ReAl ACAdemia Española (2010): Diccionario de la lengua española. Versión online: $<$ http://buscon.rae.es/drae/>.

RYAN, Chris/CAVE, Jenny (2005): "Structuring destination image: a qualitative approach", en: Journal of Travel Research 44, 2, 143-150.

Sager, Juan C./Dungworth, David/McDonald, Peter F. (1980): English Special Languages: Principles and Practice in Science and Technology. Wiesbaden: Oscar Brandstetter.

VARgas Sierra, Chelo (2005): Aproximación terminográfica al lenguaje de la piedra natural. Propuesta de sistematización para la elaboración de un diccionario traductológico. Tesis doctoral de la Universidad de Alicante, Facultad de Filosofia y Letras, Departamento de Filología Inglesa.

\section{Páginas electrónicas consultadas}

Web de hoteles Muchoviaje.com: <http://hoteles.muchoviaje.com/hoteles/hotel-hotelrural-el-rosal-en-cuenca-espana_ho667492.aspx $>$.

Web Marina d'Or (2011): <http://www.marinador.com>.

WebTaller.com (2011): <http://www.webtaller.com/maletin/articulos/reglas_de_oro_ del_desarrollo_web.php $>$. 